\title{
Poster: When Autonomous Drones Meet Driverless Cars
}

\author{
Qing Wang \\ KU Leuven \\ Leuven, Belgium \\ qing.wang@kuleuven.be
}

\author{
Chenren $\mathrm{Xu}$ \\ Peking University \\ Beijing, China \\ chenren@pku.edu.cn
}

\author{
Supeng Leng \\ UESTC \\ Chengdu, China \\ spleng@uestc.edu.cn
}

Sofie Pollin

KU Leuven

Leuven, Belgium

sofie.pollin@kuleuven.be

\begin{abstract}
In this poster, we envision the promising cooperation between autonomous drones and driverless cars. We discuss potential applications and opportunities enabled by this cooperation.
\end{abstract}

\section{CCS CONCEPTS}

- Networks $\rightarrow$ Mobile networks;

\section{ACM Reference Format:}

Qing Wang, Chenren Xu, Supeng Leng, and Sofie Pollin. 2018. Poster: When Autonomous Drones Meet Driverless Cars. In MobiSys '18: The 16th Annual International Conference on Mobile Systems, Applications, and Services, June 10-15, 2018, Munich, Germany. ACM, New York, NY, USA, 1 page. https: //doi.org/10.1145/3210240.3210806

\section{INTRODUCTION}

Driverless cars are revolutionizing our traditional driving by removing the input from people and improving the driving security and efficiency. Driverless cars have been able to fuse many sensors, e.g. camera, Lidar, mmWave radar, to sense their surroundings. Cooperation between platoon/neighboring cars through vehicular/cellular networks is also been studied to enable further sharing of critical information such as road conditions ahead. Meanwhile, autonomous drones are being investigated a lot by both academia and industry, e.g. OmniDrone [1], Skydio [2]. Powered by complex hardware and advanced techniques and Artificial Intelligence, autonomous drones are expected to enable a manifold of promising services, with applications ranging from 3D modeling, surveillance of large areas, search and rescue, and aerial delivery.

However, both driverless cars and autonomous drones are still facing their own problems. The former is being equipped with increasing number of sensors that boost the system's complex and therefore, could degrade its stability performance. While cooperating among neighboring cars can alleviate the dependency on their own sensors, this cooperation is costly, e.g. to achieve bird's eyes view. For the latter, a big challenge is their limited on-board resources, e.g. power and computing ability, that prevent drones from traveling far away and discourage them from hosting complex computing which is indispensable in efficient autonomous flying.

When autonomous drones and driverless cars are in action, many applications/benefits can be enabled, as shown in Fig. 1.

Permission to make digital or hard copies of part or all of this work for personal or classroom use is granted without fee provided that copies are not made or distributed for profit or commercial advantage and that copies bear this notice and the full citation on the first page. Copyrights for third-party components of this work must be honored

For all other uses, contact the owner/author(s).

MobiSys '18, fune 10-15, 2018, Munich, Germany

(c) 2018 Copyright held by the owner/author(s).

ACM ISBN 978-1-4503-5720-3/18/06.

https://doi.org/10.1145/3210240.3210806

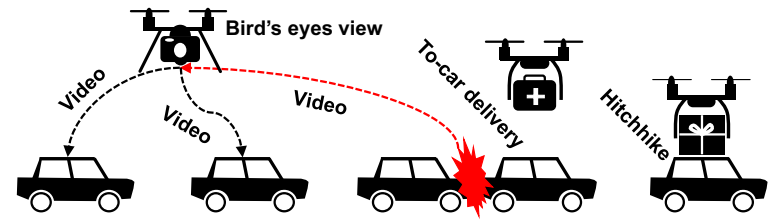

Figure 1: Autonomous drones and cars in action

- Bird's eyes view for driverless cars. Cooperating the cars in a platoon to obtain bird's eyes view consumes many resources. With the inputs from flying drones in proximity, this aim can be easily achieved with much lower resource consumption.

- Hitchhike for autonomous drones. Due to their limited power resource, drones cannot fly for long distances. If they can hitchhike on the cars that are moving on the same direction, they will save lots of energy and expand their working areas greatly.

- To-car delivery. In the era of self-driving, we will have more time when in the car and can enjoy shoppings. Autonomous drones can delivery the goods to our self-driving cars.

\section{RESEARCH OPPORTUNITIES}

There are many opportunities rising from the cooperation of autonomous drones and driverless cars. To name a few:

Inter drone-car task offloading. Study when and what tasks can be offloaded from autonomous drones to driverless cars or an edge, and vice verse. For instance, offloading resource-immediate computing tasks from drones to cars, and resource-intensity computing tasks to the edge [3]. We are currently working on designing a framework for the task offloading.

Inter drone-car high speed communication. Study how to exchange data efficiently between cars and drones considering the mobility and the density of vehicles. We have experiences with ground-to-air communication [4], and now are exploiting visible light communication to improve the communication performance.

Route plan. Study how to dynamically navigate the cars and drones based on their destinations, real-time on-board available resources, road conditions, among others.

Cooperation incentive. Study how to incentive the cooperation between autonomous drones and cars, in order to optimize the performance of the individuals as well as the whole network. Game theory could be applied here.

Novel applications. Investigate more the potential useful applications enabled/enhanced by this novel cooperation.

\section{REFERENCES}

[1] OmniDrone. https://www.omnidrone720.com/.

[2] Skydio. https://www.skydio.com/.

[3] 5GAA. Toward full connected vehicles: Edge computing for advanced automotive communications. While paper (2017).

[4] Azari, M., Rosas, F., And Pollin, S. Reshaping cellular networks for the sky: The major factors and feasibility. In IEEE ICC (2018). 